\title{
Importance of diffraction in determining the dispersion of designer surface plasmons
}

\author{
E. Hendry, * A. P. Hibbins, and J. R. Sambles \\ Electromagnetic Materials Group, School of Physics, University of Exeter, Stocker Road, Exeter EX4 4QL, United Kingdom
}

(Received 21 April 2008; revised manuscript received 27 July 2008; published 19 December 2008)

\begin{abstract}
By employing a modified modal matching approach, we obtain explicit analytical expressions relating frequency to in-plane wave vector for the surface electromagnetic mode confined at the interface between vacuum and a perfect conductor patterned with a two-dimensional square array of square holes. Our complete analytical formalism takes into account both multiple order waveguide modes and diffracted evanescent waves, hence overcoming the a priori assumptions intrinsic to previous descriptions of the dispersion of these surface waves. We validate our derived dispersion relation through comparison with that recently recorded at microwave frequencies using prism coupling. Finally, we show that diffracted evanescent waves play an important role in determining the dispersion, so that the electric field associated with "designer" surface modes is much more weakly confined to the interface than the field associated with surface plasmons on real metal surfaces.
\end{abstract}

DOI: $10.1103 /$ PhysRevB.78.235426

PACS number(s): 73.20. $-\mathrm{r}, 42.25 . \mathrm{Bs}, 42.79 .-\mathrm{e}, 78.68 .+\mathrm{m}$

\section{INTRODUCTION}

The electromagnetic response of metals at low frequencies is dominated by their large conductivities, making it difficult to exploit the unique properties of surface plasmons (SPs) at wavelengths beyond the infrared. However, it has been known for some decades that perforated and structured metallic interfaces can guide terahertz ${ }^{1}$ and microwave radiation. ${ }^{2}$ More recently, the exciting realization that one can design metal-dielectric composites to obtain unique SPlike properties has opened the field of plasmonics to lowfrequency domains. Pendry and co-workers ${ }^{3,4}$ were among the first to speculate about an extension of plasmonics into these long-wavelength regimes using these new composite materials. Pendry and co-workers ${ }^{3,4}$ proposed that even in the perfectly conducting limit (i.e., using materials with infinite conductivity), a perforated surface of a highly conducting substrate can support SP-like interface modes, and formulated an analytical dispersion equation for the mode. The dispersion of the mode asymptotically approaches a cutoff limit, characteristic of the holes, representing the effective surface plasma frequency. This result highlighted the possibility of creating "designer" (or "spoof") SP-like modes with almost arbitrary dispersion generated through structure rather than material composition. The existence of these surface modes has since been confirmed by rigorous numerical modeling $^{5-8}$ and experiments at microwave ${ }^{9}$ and, subsequently terahertz frequencies. ${ }^{10}$

A structure similar to that considered by Pendry and co-workers ${ }^{3,4}$ in their analytical approach is depicted in Fig. 1. It consists of a metallic surface (considered to be perfectly conducting) into which is impressed rectangular holes with sides $a_{1}$ and $a_{2}$ in a rectangular array described by a unit cell with sides $d_{1}$ and $d_{2}$ (note that the authors in Refs. 3 and 4 considered the special case of a square array, i.e., where $a$ $\equiv a_{1}=a_{2}$ and $d \equiv d_{1}=d_{2}$ ). This structure is similar to, but much thicker than, that analyzed by Ulrich and Tacke ${ }^{1}$ in their earlier work, with an addition of a lossless dielectric material described by relative dielectric constant $\varepsilon_{h}$ and a relative permeability of unity inside the cavities. This dielectric material plays an important role in determining the prop- erties of the surface, as it reduces the cutoff frequency for light propagating in the holes. The holes may be considered as waveguides having a cutoff frequency below which only evanescent fields exist on the metal side of the interface. It is precisely this characteristic that is required for a localized surface mode to be supported. In order to determine the dispersion relation of the SP-like modes on a surface penetrated by infinitely deep holes, Pendry and co-workers ${ }^{3,4}$ considered the field inside and outside the holes to be approximated by the $\mathrm{TE}_{s t}=\mathrm{TE}_{01}$ waveguide mode (i.e., the least strongly evanescent for frequencies below cutoff) and the specular reflection of the surface, respectively. The resulting analytical dispersion relations derived in Refs. 3 and 4 by Pendry and co-workers are therefore considered only in the limit $a \ll d$ $\ll \lambda_{0}$, where $\lambda_{0}$ is the vacuum wavelength. Moreover, even in this "long-wavelength" regime, García de Abajo and Sáenz pointed out that significant discrepancies from the dispersion relation first predicted by Pendry and co-workers ${ }^{3,4}$ may be expected when higher order waveguide modes are included in calculations. Indeed, this was substantiated using exact numerical modeling ${ }^{5}$ for an array of infinitely deep holes with dimensions $a=0.8 d$. Further numerical modeling (using finite element, ${ }^{6}$ modal expansion, ${ }^{7}$ and finite time domain ${ }^{8}$ numerical methods) has shown that hole period and depth can be particularly critical in determining the precise dispersion relation of the surface mode supported by these substrates. It is clear that a universal analytical formalism is required.

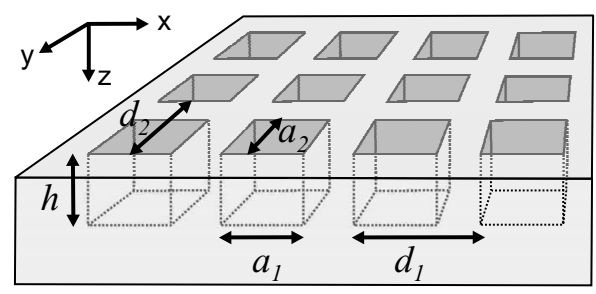

FIG. 1. Interface between vacuum and a two-dimensional, periodic array of square holes in a perfect conductor. The holes considered here are of depth $h$, side $a$, separated by distance $d$ and filled by dielectric $\varepsilon_{h}$. Unless otherwise stated, we take $\varepsilon_{h}=10$. 
Here we employ a modified modal matching method to take into account multiple order waveguide modes, diffracted evanescent waves, and finite hole depth. We overcome the $a$ priori assumptions intrinsic to the analytical formalism in Refs. 3 and 4, obtaining explicit analytical expressions relating frequency to in-plane wave vector for the electromagnetic surface mode at the interface between a vacuum and a periodically perforated perfect conductor. We validate our derived dispersion relation through comparison to the recently measured dispersion of a prism-coupled microwave SP-like surface mode. Finally, we show that diffracted evanescent waves play a very significant role in determining the dispersion that results in the confinement of designer SPs being significantly weaker than that of real SPs on flat metal surfaces.

\section{THEORETICAL FORMALISM}

Below, we develop the formalism to describe the rectangular grating in Fig. 1, based on the earlier work of McPhedran et al. ${ }^{11}$ Our starting point is to define expressions for the electric and magnetic fields in the regions either side of the interface. For simplicity, note that in the following we omit the time $(t)$ dependent component to the fields, $\sim \exp (i \omega t)$, where $\omega$ is the radial frequency. We express the electric field in the vacuum region as a two-dimensional Fourier-Floquet expansion of diffracted orders

$$
\begin{aligned}
& E_{x}^{\mathrm{vac}}=\sum_{m, n} A_{x}^{m, n} \psi_{1}(x, y) \exp \left(-i k_{z}^{m, n} z\right), \\
& E_{y}^{\mathrm{vac}}=\sum_{m, n} A_{y}^{m, n} \psi_{1}(x, y) \exp \left(-i k_{z}^{m, n} z\right),
\end{aligned}
$$

where

$$
\psi_{1}(x, y)=\exp \left[i\left(k_{x}+\frac{2 m \pi}{d_{1}}\right) x\right] \exp \left[i\left(k_{y}+\frac{2 n \pi}{d_{2}}\right) y\right] .
$$

The integers $m$ and $n$ denote the diffracted orders from the bigrating of pitch $d_{1}$ and $d_{2}$ in the $x$ and $y$ directions, respectively, and the factors $A^{m, n}$ describe the electric-field amplitude. $\boldsymbol{k}$ is the wave vector associated with a particular diffracted order. In our derivation, since we are interested in the dispersion of a propagating surface mode, we assume $k_{x}$ and $k_{y}$ to be real. The $z$ component of the wave vector can be written as

$$
k_{z}^{m, n}=\sqrt{\left(\frac{\omega}{c}\right)^{2}-\left(k_{x}+\frac{2 m \pi}{d_{1}}\right)^{2}-\left(k_{y}+\frac{2 n \pi}{d_{2}}\right)^{2}},
$$

where $c$ is the speed of light. Inside the holes, the electric field is represented by the modes of the cavity,

$$
E_{x}^{\mathrm{cav}}=\sum_{s, t}\left[B_{x}^{s, t} \psi_{2}(x, y) \exp \left(i q_{z}^{s, t} z\right)+C_{x}^{s, t} \psi_{2}(x, y) \exp \left(-i q_{z}^{s, t} z\right)\right],
$$

$$
E_{y}^{\mathrm{cav}}=\sum_{s, t}\left[B_{y}^{s, t} \psi_{3}(x, y) \exp \left(i q_{z}^{s, t} z\right)+C_{y}^{s, t} \psi_{3}(x, y) \exp \left(-i q_{z}^{s, t} z\right)\right]
$$

where

$$
\begin{aligned}
& \psi_{2}(x, y)=\cos \left(\frac{s \pi x}{a_{1}}\right) \sin \left(\frac{t \pi y}{a_{2}}\right), \\
& \psi_{3}(x, y)=\sin \left(\frac{s \pi x}{a_{1}}\right) \cos \left(\frac{t \pi y}{a_{2}}\right) .
\end{aligned}
$$

The positive integers $s$ and $t$ denote the waveguide mode, and the factors $B^{s, t}$ and $C^{s, t}$ describe the electric-field amplitudes of the decaying wave in the cavity and the reflected wave from the cavity bottom, respectively. Note that Eqs. (3c) and (3d) represent both TE and TM polarized modes. The propagation constant associated with a particular mode can be written as

$$
q_{z}^{s, t}=\sqrt{\varepsilon_{h}\left(\frac{\omega}{c}\right)^{2}-\left(\frac{s \pi}{a_{1}}\right)^{2}-\left(\frac{t \pi}{a_{2}}\right)^{2}}
$$

This quantity is dependent on the dielectric constant of the material inside the hole. To aid discussion in the following sections, unless otherwise stated, we choose a value of $\varepsilon_{h}$ $=10$ for this material. By noting that the electric field at the bottom of a cavity (i.e., at $z=h$ ) in a perfect electrical conductor (PEC) must be zero, we can simplify Eqs. (3a) and (3b), obtaining

$$
\begin{aligned}
& E_{x}^{\mathrm{cav}}=\sum_{s, t} B_{x}^{s, t} \psi_{2}(x, y)\left\{\exp \left(i q_{z}^{s, t} z\right)-\exp \left[i q_{z}^{s, t}(2 h-z)\right]\right\} \\
& E_{y}^{\mathrm{cav}}=\sum_{s, t} B_{y}^{s, t} \psi_{3}(x, y)\left\{\exp \left(i q_{z}^{s, t} z\right)-\exp \left[i q_{z}^{s, t}(2 h-z)\right]\right\}
\end{aligned}
$$

We can obtain the $z$ components of the electric field inside and outside the cavity, and subsequently expressions for the magnetic field $\boldsymbol{H}$, through the free space Maxwell's relations $\boldsymbol{\nabla} \cdot \boldsymbol{E}=0$ and $\boldsymbol{\nabla} \times \boldsymbol{E}=-\mu_{0} \partial \boldsymbol{H} / \partial t$. In the vacuum region, the resulting $x$ and $y$ components of $\boldsymbol{H}$ have the form

$$
H_{x}^{\mathrm{vac}}=-\sum_{m, n} \frac{A_{x}^{m, n}\left(k_{x}+\frac{2 m \pi}{d_{1}}\right)\left(k_{y}+\frac{2 n \pi}{d_{2}}\right)+A_{y}^{m, n}\left[\left(k_{z}^{m, n}\right)^{2}+\left(k_{y}+\frac{2 n \pi}{d_{2}}\right)^{2}\right]}{i \omega \mu_{0} k_{z}^{m, n}} \psi_{1}(x, y) \exp \left(-i k_{z}^{m, n} z\right),
$$




$$
H_{y}^{\mathrm{vac}}=\sum_{m, n} \frac{A_{y}^{m, n}\left(k_{x}+\frac{2 m \pi}{d_{1}}\right)\left(k_{y}+\frac{2 n \pi}{d_{2}}\right)+A_{x}^{m, n}\left[\left(k_{z}^{m, n}\right)^{2}+\left(k_{x}+\frac{2 m \pi}{d_{1}}\right)^{2}\right]}{i \omega \mu_{0} k_{z}^{m, n}} \psi_{1}(x, y) \exp \left(-i k_{z}^{m, n} z\right),
$$

while in the cavity

$$
\begin{aligned}
H_{x}^{\mathrm{cav}}= & \sum_{s, t} \frac{B_{x}^{s, t} \frac{\pi^{2} s t}{a_{1} a_{2}}+B_{y}^{s, t}\left[\left(q_{z}^{s, t}\right)^{2}+\left(\frac{\pi t}{a_{2}}\right)^{2}\right]}{i \mu_{0} \omega q_{z}^{s, t}} \psi_{3}(x, y) \\
& \times\left\{\exp \left(i q_{z}^{s, t} z\right)+\exp \left[i q_{z}^{s, t}(2 h-z)\right]\right\}, \\
H_{y}^{\mathrm{cav}}= & -\sum_{s, t} \frac{B_{y}^{s, t} \frac{\pi^{2} s t}{a_{1} a_{2}}+B_{x}^{s, t}\left[\left(q_{z}^{s, t}\right)^{2}+\left(\frac{\pi s}{a_{1}}\right)^{2}\right]}{i \mu_{0} \omega q_{z}^{s, t}} \psi_{2}(x, y) \\
& \times\left\{\exp \left(i q_{z}^{s, t} z\right)+\exp \left[i q_{z}^{s, t}(2 h-z)\right]\right\} .
\end{aligned}
$$

We now have the $x$ and $y$ components of the electric and magnetic fields in both regions in terms of the set of unknowns $A_{x}^{m, n}, A_{y}^{m, n}, B_{x}^{s, t}$, and $B_{y}^{s, t}$. In order to eliminate these unknowns, we use the fact that both the $x$ and $y$ components of the electric field must be continuous at the vacuumsubstrate interface (i.e., $z=0$ ) over the entire unit cell, while the magnetic-field components are continuous only at the hole aperture. Matching Eqs. (1a) and (5a) and Eqs. (1b) and (5b), multiplying by $\psi_{1}^{*}(x, y)$ and integrating over $x$ and $y$ from 0 to $d_{1}$ and 0 to $d_{2}$, respectively, taking into account the orthogonality of the eigenmodes of the system, yields

$$
d_{1} d_{2} A_{x}^{m, n}=\sum_{s, t} B_{x}^{s, t}\left[1-\exp \left(i 2 q_{z}^{s, t} h\right)\right] R_{1}^{s, t, m, n},
$$

$$
d_{1} d_{2} A_{y}^{m, n}=\sum_{s, t} B_{y}^{s, t}\left[1-\exp \left(i 2 q_{z}^{s, t} h\right)\right] R_{2}^{s, t, m, n},
$$

where

$$
\begin{aligned}
R_{1}^{s, t, m, n}= & \int_{0}^{a_{2}} \int_{0}^{a_{1}} \cos \left(\frac{s \pi x}{a_{1}}\right) \sin \left(\frac{t \pi y}{a_{2}}\right) \\
& \times \exp \left[-i\left(k_{x}+\frac{2 m \pi}{d_{1}}\right) x\right] \\
& \times \exp \left[-i\left(k_{y}+\frac{2 n \pi}{d_{2}}\right) y\right] d x d y, \\
R_{2}^{s, t, m, n}= & \int_{0}^{a_{2}} \int_{0}^{a_{1}} \sin \left(\frac{s \pi x}{a_{1}}\right) \cos \left(\frac{t \pi y}{a_{2}}\right) \\
& \times \exp \left[-i\left(k_{x}+\frac{2 m \pi}{d_{1}}\right) x\right] \\
& \times \exp \left[-i\left(k_{y}+\frac{2 n \pi}{d_{2}}\right) y\right] d x d y .
\end{aligned}
$$

From Eqs. (8a) and (8b) we see that we have obtained expressions relating the unknowns $A_{x}^{m, n}$ and $A_{y}^{m, n}$ in terms of the unknowns $B_{x}^{s, t}$ and $B_{y}^{s, t}$. We obtain similar expressions by multiplying equation pairs Eqs. (6a) and (7a) and Eqs. (6b) and (7b) by $\psi_{3}(x, y)$ and $\psi_{2}(x, y)$, respectively, and integrating from 0 to $a_{1}$ and 0 to $a_{2}$ for $x$ and $y$, respectively,

$$
\begin{gathered}
-\frac{a_{1} a_{2}[1+\delta(t)]}{4} \frac{B_{x}^{s, t} \frac{\pi^{2} s t}{a_{1} a_{2}}+B_{y}^{s, t}\left[\left(q_{z}^{s, t}\right)^{2}+\left(\frac{\pi t}{a_{2}}\right)^{2}\right]}{q_{z}^{s, t}}\left[1+\exp \left(i 2 q_{z}^{s, t} h\right)\right] \\
=\sum_{m, n} \frac{A_{x}^{m, n}\left(k_{x}+\frac{2 m \pi}{d_{1}}\right)\left(k_{y}+\frac{2 n \pi}{d_{2}}\right)+A_{y}^{m, n}\left[\left(k_{z}^{m, n}\right)^{2}+\left(k_{y}+\frac{2 n \pi}{d_{2}}\right)^{2}\right]}{k_{z}^{m, n}} R_{3}(m, n, s, t), \\
-\frac{a_{1} a_{2}[1+\delta(s)]}{4} \frac{B_{y}^{s, t} \frac{\pi^{2} s t}{a_{1} a_{2}}+B_{x}^{s, t}\left[\left(q_{z}^{s, t}\right)^{2}+\left(\frac{\pi s}{a_{1}}\right)^{2}\right]}{q_{z}^{s, t}}\left[1+\exp \left(i 2 q_{z}^{s, t} h\right)\right] \\
=\sum_{m, n} \frac{A_{y}^{m, n}\left(k_{x}+\frac{2 m \pi}{d_{1}}\right)\left(k_{y}+\frac{2 n \pi}{d_{2}}\right)+A_{x}^{m, n}\left[\left(k_{z}^{m, n}\right)^{2}+\left(k_{x}+\frac{2 m \pi}{d_{1}}\right)^{2}\right]}{k_{z}^{m, n}} R_{4}(m, n, s, t),
\end{gathered}
$$


where

$$
\begin{aligned}
R_{3}^{s, t, m, n}= & \int_{0}^{a_{2}} \int_{0}^{a_{1}} \sin \left(\frac{s \pi x}{a_{1}}\right) \cos \left(\frac{t \pi y}{a_{2}}\right) \\
& \times \exp \left[i\left(k_{x}+\frac{2 m \pi}{d_{1}}\right) x\right] \exp \left[i\left(k_{y}+\frac{2 n \pi}{d_{2}}\right) y\right] d x d y \\
R_{4}^{s, t, m, n}= & \int_{0}^{a_{2}} \int_{0}^{a_{1}} \cos \left(\frac{s \pi x}{a_{1}}\right) \sin \left(\frac{t \pi y}{a_{2}}\right) \\
& \times \exp \left[i\left(k_{x}+\frac{2 m \pi}{d_{1}}\right) x\right] \exp \left[i\left(k_{y}+\frac{2 n \pi}{d_{2}}\right) y\right] d x d y
\end{aligned}
$$

combining Eqs. (8a) and (8b) and Eqs. (9a) and (9b) and eliminating $A_{x}^{m, n}$ and $A_{y}^{m, n}$, we find two sets of coupled linear equations that contain the unknowns $B_{x}^{s, t}$ and $B_{y}^{s, t}$,

$$
\begin{aligned}
& \sum_{s, t}\left[Q_{1}\left(s, t, s^{\prime}, t^{\prime}\right) B_{x}^{s, t}+Q_{2}\left(s, t, s^{\prime}, t^{\prime}\right) B_{y}^{s, t}\right]=0, \\
& \sum_{s, t}\left[Q_{3}\left(s, t, s^{\prime}, t^{\prime}\right) B_{x}^{s, t}+Q_{4}\left(s, t, s^{\prime}, t^{\prime}\right) B_{y}^{s, t}\right]=0,
\end{aligned}
$$

and where $\delta$ represents the Kronecker delta function. By

where the coefficients $Q$ are given by

$$
\begin{aligned}
& Q_{1}=\sum_{m, n}\left[\frac{R_{1}^{m, n, s, t} R_{3}^{m, n, s^{\prime}, t^{\prime}}\left(k_{x}+\frac{2 m \pi}{d_{1}}\right)\left(k_{y}+\frac{2 n \pi}{d_{2}}\right)}{k_{z}^{m, n}} \frac{1-\exp \left(i 2 q_{z}^{s, t} h\right)}{1+\exp \left(i 2 q_{z}^{s^{\prime}, t^{\prime}} h\right)}\right]+\delta\left(t-t^{\prime}\right) \delta\left(s-s^{\prime}\right) \frac{a_{1} a_{2} d_{1} d_{2}\left[1+\delta\left(t^{\prime}\right)\right]}{4 q_{z}^{s^{\prime}, t^{\prime}}} \frac{\pi^{2} s^{\prime} t^{\prime}}{a_{1} a_{2}}, \\
& Q_{2}=\sum_{m, n}\left\{\frac{R_{2}^{m, n, s, t} R_{3}^{m, n, s^{\prime}, t^{\prime}}\left[\left(k_{z}^{m, n}\right)^{2}+\left(k_{y}+\frac{2 n \pi}{d_{2}}\right)^{2}\right]}{k_{z}^{m, n}} \frac{1-\exp \left(i 2 q_{z}^{s, t} h\right)}{1+\exp \left(i 2 q_{z}^{s^{\prime}, t^{\prime}} h\right)}\right\}+\delta\left(t-t^{\prime}\right) \delta\left(s-s^{\prime}\right) \frac{a_{1} a_{2} d_{1} d_{2}\left[1+\delta\left(t^{\prime}\right)\right]}{4 q_{z}^{s^{\prime}, t^{\prime}}} \\
& \times\left[\left(q_{z}^{s^{\prime}, t^{\prime}}\right)^{2}+\left(\frac{\pi t^{\prime}}{a_{2}}\right)^{2}\right], \\
& Q_{3}=\sum_{m, n}\left\{\frac{R_{1}^{m, n, s, t} R_{4}^{m, n, s^{\prime}, t^{\prime}}\left[\left(k_{z}^{m, n}\right)^{2}+\left(k_{x}+\frac{2 m \pi}{d_{1}}\right)^{2}\right]}{k_{z}^{m, n}} \frac{1-\exp \left(i 2 q_{z}^{s, t} h\right)}{1+\exp \left(i 2 q_{z}^{s^{\prime}, t^{\prime}} h\right)}\right\}+\delta\left(t-t^{\prime}\right) \delta\left(s-s^{\prime}\right) \frac{a_{1} a_{2} d_{1} d_{2}\left[1+\delta\left(s^{\prime}\right)\right]}{4 q_{z}^{s^{\prime}, t^{\prime}}} \\
& \times\left[\left(q_{z}^{s^{\prime}, t^{\prime}}\right)^{2}+\left(\frac{\pi s^{\prime}}{a_{1}}\right)^{2}\right], \\
& Q_{4}=\sum_{m, n}\left[\frac{R_{2}^{m, n, s, t} R_{4}^{m, n, s^{\prime}, t^{\prime}}\left(k_{x}+\frac{2 m \pi}{d_{1}}\right)\left(k_{y}+\frac{2 n \pi}{d_{2}}\right)}{k_{z}^{m, n}} \frac{1-\exp \left(i 2 q_{z}^{s, t} h\right)}{1+\exp \left(i 2 q_{z}^{s^{\prime}, t^{\prime}} h\right)}\right]+\delta\left(t-t^{\prime}\right) \delta\left(s-s^{\prime}\right) \frac{a_{1} a_{2} d_{1} d_{2}\left[1+\delta\left(s^{\prime}\right)\right]}{4 q_{z}^{s^{\prime}, t^{\prime}}} \frac{\pi^{2} s^{\prime} t^{\prime}}{a_{1} a_{2}} .
\end{aligned}
$$

Note that the dummy variables $s^{\prime}$ and $t^{\prime}$ are introduced in place of $s$ and $t$ in Eqs. (9a) and (9b) before combining with Eqs. (8a) and (8b) in order to achieve a complete set of equations in Eq. (10). This concludes the formalism of the problem. We solve the coupled Eq. (10) in the normal fashion by finding

$$
\operatorname{Det}(\mathbf{M})=0,
$$

where $\mathbf{M}$ is a square matrix with the elements in each column of $\mathbf{M}$ representing the coefficients $Q$ of the unknowns
$B_{x}^{s, t}$ and $B_{y}^{s, t}$, while the coefficients in a particular row are specified by the positive integer values $s^{\prime}$ and $t^{\prime}$. Equation (12) then gives a relationship between frequency and wave vector, i.e., we have found the dispersion relation of the system.

\section{RESULTS AND DISCUSSION}

\section{A. Significance of terms in the dispersion relation}

In principle, in solving Eq. (12) we can include any number of waveguide modes and/or diffracted orders, i.e., our 
solution is complete, without the inherent approximations of the analytical formalisms presented in Refs. 3 and 4. However, it is more informative to consider the components of matrix $\mathbf{M}$ that have the largest effect on the dispersion of the surface mode. Note that higher order diffraction and waveguide modes may each be introduced separately. In this section, we do this by sequentially introducing higher order terms into matrix $\mathbf{M}$ and finding the resulting dispersion. We then show that the importance of higher order terms and the mathematical form of the dispersion relation is very much dependent on the structural dimensions (i.e., $a_{1}, a_{2}, d_{1}, d_{2}$, and $h$ ). For simplicity, we restrict ourselves in the remaining sections below to the discussion of the symmetric case introduced in Refs. 3-5, given by $a \equiv a_{1}=a_{2}$ and $d \equiv d_{1}=d_{2}$.

The simplest solution to consider is for infinitely deep holes $(h \rightarrow \infty)$, taking into account only the specular diffracted beam [i.e., consider only $m=n=0$ for the coefficients in Eqs. (10)] and only the $\mathrm{TE}_{01}$ waveguide mode [i.e., consider only the mode defined by $(s=0, t=1)$ in Eqs. (10)]. This results in a simple $2 \times 2$ matrix with two solutions for Eq. (12). The first of these, being the solution to $\left(q_{z}^{0,1}\right)^{2}$ $+(\pi / a)^{2}=0$, is trivial, while the second gives the dispersion relation

$$
-\left(k_{0}^{2}-k_{y}^{2}\right) \frac{\left(S^{0,0}\right)^{2}}{k_{z}^{0,0} q_{z}^{0,1}}=1,
$$

where $k_{0}=\omega / c$. In Ref. $4 S^{m, n}=S^{0,0}$ is termed the "overlap integral" between the $\mathrm{TE}_{01}$ waveguide mode inside the cavity and the $(m=n=0)$ diffracted order outside the cavity, and is given by

$$
S^{0,0}=\sqrt{\frac{2}{a^{2} d^{2}} R_{1}^{0,0,0,1} R_{4}^{0,0,0,1}}=\frac{4 \pi \sqrt{2}}{a^{2} d} \frac{\sin \left(a k_{x} / 2\right) \cos \left(a k_{y} / 2\right)}{k_{x}\left[(\pi / a)^{2}-\left(k_{y}\right)^{2}\right]} .
$$

We note that Eq. (13) gives precisely the dispersion relation obtained by Pendry and co-workers ${ }^{3,4}$ for $k_{y}=0$, i.e., for dispersion in the $x$ direction of the sample. The cutoff frequency of the holes, given by the root of $q_{x}^{1,0}$, is characterized by the quantization condition that the tangential electric field must be zero at the walls of the cavity, and amounts to

$$
\omega_{a}^{\infty}=\frac{\pi c}{a \sqrt{\varepsilon_{h}}} .
$$

For normalization purposes below, it is convenient to also define the corresponding free space wave vector,

$$
k_{a}^{\infty}=\frac{\omega_{a}^{\infty}}{c} .
$$

For such infinitely deep holes, the condition in Eq. (15) defines a frequency below which no propagating fields are allowed inside the cavity, and so only evanescent fields exist on the metal side for frequencies below this limit. It is exactly this evanescent field characteristic that is required for the support of a localized surface mode. The dispersion of the mode asymptotically approaches this cutoff frequency, which represents the effective surface plasma frequency of the substrate.
For holes of finite depth $h$ formed in a perfect conductor, the electric field must decay to zero at the bottom of the cavity $(z=h)$. This restriction of finite depth places an additional quantization condition on the waveguide mode in the $z$ direction, i.e., the hole cutoff is determined by both their breadth and depth. One can therefore expect this additional quantization to modify the effective surface plasma frequency of the substrate. We note that an earlier attempt at finding an analytical solution to the finite depth problem ${ }^{4}$ was derived in the long-wavelength limit (i.e., $d \ll \lambda_{0}$ ). Here, we make no such assumptions. Indeed it is straightforward to extend our consideration above to holes of finite depth $h$, obtaining

$$
\left(k_{0}^{2}-k_{y}^{2}\right)^{2} \frac{i \tan \left(h q_{z}^{0,1}\right)\left(S^{0,0}\right)^{2}}{k_{z}^{0,0} q_{z}^{0,1}}=1,
$$

i.e., the left-hand side of the relation is modified by the finite depth of the holes giving rise to an $i \tan \left(l q_{z}^{0,1}\right)$ term which accounts for the quantization of electric field in the $z$ direction. This term modifies the asymptotic frequency of the dispersion, given by the root of $q_{x}^{1,0} \cot \left(h q_{x}^{1,0}\right)$,

$$
\omega_{a}=\frac{\pi c}{\sqrt{\varepsilon_{h}}} \sqrt{\frac{1}{a^{2}}+\frac{1}{4 h^{2}}} .
$$

Since the electric field should be a maximum at the entrance and zero at the bottom of the cavity, the lowest energy quantized mode involves a quarter of one wavelength, hence the factor inside the square root of Eq. (18).

One of the principal approximations in the derivation above (and in Refs. 3 and 4) is made by considering only the $\mathrm{TE}_{01}$ (i.e., $s=0, t=1$ ) waveguide mode in describing the field inside the cavities. García de Abajo and Sáenz ${ }^{5,12}$ demonstrated that the inclusion of higher order waveguide modes generates a dispersion relation for an array of infinitely deep holes which is very different in the long-wavelength limit (i.e., $a \ll d \ll \lambda_{0}$ ) from that predicted by Pendry and co-workers. ${ }^{3,4}$ However, in the region of interest here, near the cutoff frequency of the cavities, the dispersion will predominantly be determined by the near resonant $\mathrm{TE}_{01}$ waveguide mode. The higher order waveguide modes with the correct symmetry (e.g., the $\mathrm{TE}_{03}$ ) are very strongly evanescent [i.e., in Eq. (4), $q_{z}^{s, t}$ is large and imaginary] when compared to the resonant $\mathrm{TE}_{01}$ mode, and will therefore have little effect on the surface mode dispersion near the cutoff frequency of the cavities. It can easily be shown by truncating matrix $\mathbf{M}$ at different points and numerically solving Eq. (12) that the inclusion of higher order waveguide modes in our formulation does not significantly perturb the dispersion of the surface mode for the frequencies plotted in Fig. 2. The effect of including higher order waveguide modes in the long-wavelength limit has been extensively discussed in Refs. 5 and 12 and will not be discussed further here.

However, Pendry and co-workers ${ }^{3,4}$ also made approximations for the field outside the holes. In Refs. 3 and 4 the authors consider only the specular field outside the cavity (i.e., $m=n=0$ ). From Eq. (2), one can immediately see that this is a reasonable assumption in the long-wavelength limit, as for nonzero $m$ and $n k_{z}^{m, n}$ is large and imaginary in the 

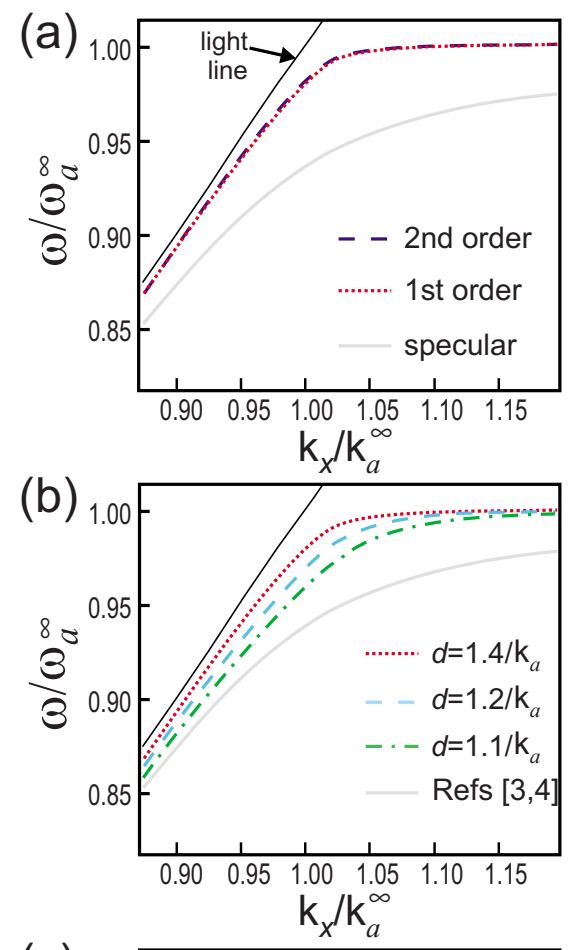

limit $\omega \rightarrow 0$. These strongly evanescent, higher order diffracted modes will therefore contribute only very minor perturbations to the dominant (and weakly evanescent) $m=n$ $=0$ mode. However, evanescent diffracted fields will be more important in determining dispersion for higher frequencies, as $k_{z}^{m, n} \rightarrow 0$ (at the Brillouin-zone boundaries). Until this point we have assumed that the fields in the vacuum region result entirely from the specular reflection of the sample. It is, however, straightforward to include higher order diffraction in our derivation of the dispersion relation, resulting in a sum over terms which have a form similar to Eq. (17),

$$
\sum_{m, n}\left[k_{0}^{2}-\left(k_{y}+\frac{2 n \pi}{d}\right)^{2}\right] \frac{i \tan \left(h q_{z}^{0,1}\right)\left(S^{m, n}\right)^{2}}{k_{z}^{m, n} q_{z}^{0,1}}=1 .
$$

The overlap integral between the $\mathrm{TE}_{01}$ waveguide mode inside the cavity and the $(m, n)$ th diffracted order outside the cavity is given by

$$
S^{m, n}=\frac{4 \pi \sqrt{2}}{a^{2} d} \frac{\sin \left[a\left(k_{x} / 2+m \pi / d\right)\right] \cos \left[a\left(k_{y} / 2+n \pi / d\right)\right]}{\left(k_{x}+2 m \pi / d\right)\left[(\pi / a)^{2}-\left(k_{y}+2 n \pi / d\right)^{2}\right]} .
$$

The dispersion given by Eq. (19) is valid near the asymptotic frequency for square arrays of holes of any size and depth. It should be noted that, in the limit that we consider only the specular diffracted order (i.e., $m=n=0$ ), infinitely deep holes and $k_{y}=0$ (i.e., dispersion in the $x$ direction), Eq. (19) again simplifies to the dispersion relation found in Refs. 3 and 4.

Including the evanescent diffracted orders in the vacuum region modifies the curvature of the dispersion in the region near the cutoff frequency. This effect is highlighted in Fig. 2(a), where we plot the dispersion relation for a sample defined by $d=1.4 / k_{a}^{\infty}, h=\infty$ and $\varepsilon_{h}=10$, with $k_{y}=0$, by first including only specular reflection [Eq. (13)], and subsequently including both first- and second-order diffraction [Eq. (19)]. Evanescent diffracted orders are evidently important even well away from the first Brillouin-zone boundary: for a surface defined by the dimensions $d=1.4 / k_{a}^{\infty}$ and $h=\infty$, the Brillouin-zone boundary occurs at $k_{x} / k_{a}^{\infty}=\pi / d k_{a}^{\infty}=2.24$, well outside the $x$-axis range of Fig. 2(a). In practice, an accurate dispersion relation is obtained by including only first-order diffraction by summing the terms in Eq. (19) for $m$ and $n$ from -1 to +1 ,

$$
\sum_{m=-1}^{+1} \sum_{n=-1}^{+1}\left[k_{0}^{2}-\left(k_{y}+\frac{2 n \pi}{d}\right)^{2}\right] \frac{i \tan \left(h q_{z}^{0,1}\right)\left(S^{m, n}\right)^{2}}{k_{z}^{m, n} q_{z}^{0,1}}=1 .
$$
zone boundary occurring at $k_{x} / k_{a}^{\infty}=2.86,2.62$, and 2.24, respectively) and $\varepsilon_{h}=10$. (c) Note that in (a) and (b) the dispersion bending is not due to the proximity of the Brillouin-zone boundary-the dashed line shows the dispersion relation obtained from Eq. (21) in this work for a structure defined by $d=3.0 / k_{a}^{\infty}$, corresponding to a Brillouin-zone boundary occurring at $k_{x} / k_{a}^{\infty}=1.05$ (marked BZ in the figure). As expected, the dispersion is symmetric around the Brillouin-zone boundary. In (a), (b), and (c), the frequency and wave vector are normalized using Eqs. (15) and (16), respectively. Higher order surface modes, above the asymptotic frequency, have been left out for clarity. at $k_{x} / k_{a}^{\infty}=\pi / d k_{a}^{\infty}=2.24$. (b) The dispersion relations for a PEC surco-workers (Refs. 3 and 4) (gray solid line) for $d=1.5 / k_{a}^{\infty}$ and $\varepsilon_{h}$ $=10$. Also plotted are the dispersion relations obtained from Eq. (21) in this work (i.e., including first-order diffraction) for hole periods defined by $d=1.1 / k_{a}^{\infty}, 1.2 / k_{a}^{\infty}$, and $1.4 / k_{a}^{\infty}$ (i.e., a Brillouin-

As we show below, this simple equation gives a remarkably accurate dispersion relation without the need for numerical modeling, ${ }^{6,7}$ allowing us to simply and straightforwardly optimize the system parameters.

The position of the Brillouin-zone boundary is determined by the periodicity of the holes. In Fig. 2(b) we plot the dispersion obtained from Eq. (21) for hole periods defined by $d=1.1 / k_{a}^{\infty}, 1.2 / k_{a}^{\infty}$, and $1.4 / k_{a}^{\infty}$ (i.e., a Brillouin-zone boundary occurring at $k_{x} / k_{a}^{\infty}=2.86,2.62$, and 2.24 , respectively). 
For small values of $d$, the Brillouin-zone boundary lies at large values of $k_{x}$, and first-order diffracted waves are strongly evanescent in the region around $k_{a}^{\infty}$ [i.e., $k_{z}^{m, n}$ from Eq. (2) is large and imaginary]. Therefore, by simply decreasing the pitch of the structure, the effect of evanescent diffracted orders can be diminished, and the dispersion tends toward that derived in Refs. 3 and 4. It should be noted that, for $\varepsilon_{h}=10$, a sample defined by period $d=1.1 / k_{a}^{\infty}$ corresponds to a hole size $a=0.9 d$. Therefore, for a structure with holes filled by dielectric with $\varepsilon_{h}=10$, any further decrease in $d$ is ultimately limited by the hole size $a$. Nevertheless, it is clear that major discrepancies exist between the dispersion relation found by Pendry and co-workers ${ }^{3,4}$ and that from Eq. (21), due to the diffracted evanescent fields associated with the periodicity of the lattice.

The divergences of the dispersion lines from the light line in Figs. 2(a) and 2(b) are not due to the proximity to the Brillouin-zone boundary - the dashed line in Fig. 2(c) shows the dispersion relation obtained from Eq. (21) in this work for a structure defined by $d=3.0 / k_{a}^{\infty}$, corresponding to a Brillouin-zone boundary occurring at $k_{x} / k_{a}^{\infty}=1.05$ (marked $\mathrm{BZ}$ in the figure). As expected, a clear turning point at the Brillouin-zone boundary is observed. Note that surface modes near a Brillouin-zone boundary will be lossy due to scattering. This effect highlights the importance of the dielectric regions in the structure shown in Fig. 1: this dielectric material reduces the cutoff frequency for light propagating in the holes, thereby reducing the effects of diffraction near to the Brillouin-zone boundary which will dominate the dispersion for similar structures with no dielectric core. ${ }^{1,10}$

\section{B. Comparison with experiment}

In this section, in order to further validate our derived dispersion relation, we compare it to the dispersion of a microwave frequency designer surface plasmon. Our 350 $\times 350 \mathrm{~mm}^{2}$ sample comprises a close-packed array of hollow, square-ended brass tubes defined by lattice spacing $d$ $=9.53 \mathrm{~mm}$ and height $h=15 \mathrm{~mm}$, placed on a flat metal plate. The inner size of the tubes is defined by $a=6.96 \mathrm{~mm}$ giving, resulting in a cutoff frequency of $22 \mathrm{GHz}$ when unfilled. In order to satisfactorily separate dispersion due to the opening of the photonic band gap at the Brillouin-zone boundary from the plasmonic dispersion, we first fill the tubes with wax $\left(\varepsilon_{h}=2.29\right)$ to lower the cutoff frequency to approximately $14 \mathrm{GHz}$, and also rotate the plane of incidence by $45^{\circ}$ around the $z$ axis so that it lies along the diagonal of the unit cell (i.e., $k_{y}=k_{x}$ ).

We experimentally determine the dispersion of the surface modes using an Otto-type prism-coupling technique [see Fig. 3(a) . ${ }^{13}$ Briefly, a large wax prism, of refractive index, $n$ $=1.5$, is used to couple collimated microwave radiation on to a surface comprised of an array of holes. For $p$-polarized (transverse magnetic) incident radiation and a suitable tunnel barrier (established by spacing the prism some $t$ millimeters from the sample) with the microwave beam incident beyond the critical angle $\left(\theta_{\text {int }}>\sin ^{-1} 1 / n\right)$, the evanescent fields beyond the prism will resonantly couple to the SP-like mode on the surface of the array, and a minimum will be recorded in (a)

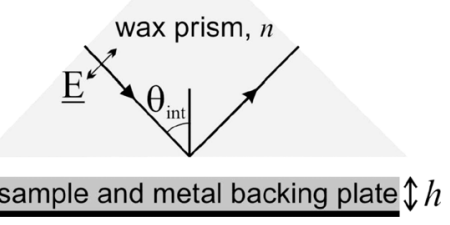

(b)

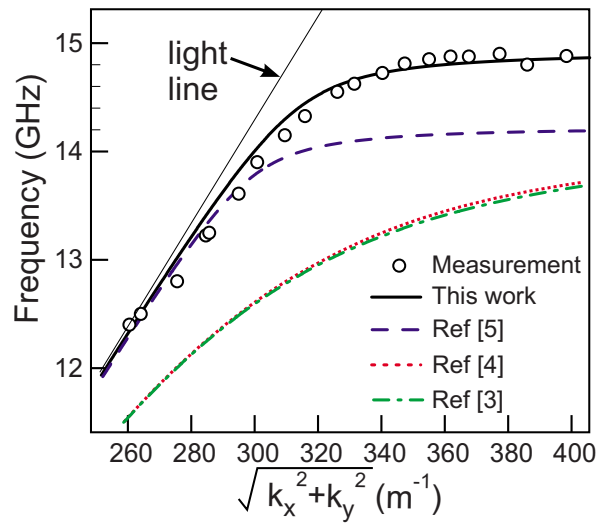

FIG. 3. (Color online) (a) Schematic of the Otto type prismcoupling experiment. (b) Real component of the dispersion relation in the diagonal direction i.e., $k_{y}=k_{x}$ ) for a sample defined by $a$ $=6.96 \mathrm{~mm}, d=9.53 \mathrm{~mm}, h=15 \mathrm{~mm}$, and $\varepsilon_{h}=2.29$. The circles represent the dispersion measured in a prism-coupling experiment. The lines represent the dispersion predicted by Pendry et al. (Ref. 3), García-Vidal et al. (Ref. 4), García de Abajo and Sáenz (Ref. 5) and by Eq. (21) in this work.

the reflected spectrum. By varying the spacing between the prism and the sample and recording the frequency of the reflectivity minimum, the in-plane wave vector of the mode may be extracted. We determine the resonant frequency at the gap spacing for which coupling to the mode is strongest, and then repeat the procedure for a number of different angles, $\theta_{\text {int }}$, allowing for a good approximation of the surface mode's dispersion to be derived. In Fig. 3(b) we compare the experimentally determined dispersion relation to our analytical dispersion from Eq. (21). We plot for comparison the dispersion relations previously published in the literature: the dispersion relations from Refs. 3 and 5 are derived for infinitely deep holes, while the analytical expression derived in Ref. 4 includes a small correction to the dispersion relation of Ref. 3 due to finite hole depth.

It is apparent from Fig. 3(b) that the dispersion relations from Refs. 3-5 do not reproduce the experimental data near the asymptotic frequency. As mentioned above, García de Abajo and Sáenz ${ }^{5,12}$ demonstrated that the inclusion of higher order waveguide modes generates a dispersion relation for an array of infinitely deep holes which, in the longwavelength limit (i.e., $a \ll d \ll \lambda_{0}$ ), lies significantly closer to the light line (and our experimentally determined dispersion relation) than that predicted by Pendry and co-workers. ${ }^{3,4}$ However, the finite length of the holes and evanescent diffracted orders need to be taken into account for the region near the asymptotic cutoff frequency: the dispersion relation given in Eq. (21) is a remarkably good fit in this region, 
accurately reproducing both the curvature and asymptote $(14.9 \mathrm{GHz})$. The inclusion of diffracted orders in our derivation clearly produces a curvature very different from that in Refs. 3 and 4.

\section{Dispersion of dimpled surfaces with dielectric cavities}

Dimpled and grooved metallic surfaces are exhibiting significant promise as substrates for confining low-frequency electromagnetic surface modes. $5,7,8,10$ We show below that while for infinitely deep holes the effect of evanescent diffractive orders perturbs the curvature of the surface mode dispersion, for surfaces perforated with shallow holes evanescent diffracted orders significantly modify both the curvature and the asymptotic frequency of the surface mode dispersion. In this section we discuss the dispersion and asymptotic frequencies of electromagnetic modes on these dimpled surfaces. In order to clearly show the effective cutoff of the holes rather than diffractive effects near the Brillouin-zone boundary we again consider a dielectric core of $\varepsilon_{h}=10$.

When higher order diffracted fields play an important role (i.e., not in the long-wavelength limit), the asymptotic frequency for the dispersion, $\omega_{a}$ and corresponding wave vector, $k_{a}$, cannot be found using Eq. (18) (i.e., the quarter wavelength standing-wave condition). Instead, we must find the asymptotic frequency by solving the dispersion relation in Eq. (21). In Fig. 4(a) we plot the dispersion obtained from Eq. (21) for a hole period defined by $d=1.1 / k_{a}^{\infty}$ (i.e., a Brillouin-zone boundary occurring at $\left.k_{x} / k_{a}^{\infty}=2.24\right)$, varying the depth of the hole, with $h=0.5 / k_{a}^{\infty}, h=1 / k_{a}^{\infty}, 2 / k_{a}^{\infty}$, and $6 / k_{a}^{\infty}$. Clearly, for small values of $h$, the asymptotic frequency of the dispersion is strongly dependent on hole depth. Similarly, for a given depth of hole, the asymptotic frequency of dispersion is also modified on changing the pitch (not shown).

The same dispersion relations are also plotted in Fig. 4(b), this time normalized by $\omega_{a}$ and $k_{a}$ for finite depth holes. ${ }^{14}$ This normalization allows us to compare the curvature of dispersion relations with very different asymptotic frequencies. It is clear from Fig. 4(b) that the dispersion of modes on shallow holed surfaces is more divergent from the light line for frequencies well below the asymptotic frequency. This is a highly desirable property, as it is this divergence from the light line that gives rise to strong confinement of the mode to the surface (see Sec. III D). From Figs. 2(b) and 4(a) one might therefore conclude that the optimum dispersion is obtained by making both $d$ and $h$ small. In practice, one will always encounter lower limits which will provide optimum values of $d$ and $h$. The first of these is trivial: $d$ cannot be smaller than the hole size $a$. For a sample with holes filled with dielectric defined by $\varepsilon_{h}=10, d=1 / k_{a}^{\infty}$ represents the lower limit for the period. The second of the lower limits is less trivial: in decreasing $h, k_{a}$ tends toward the Brillouinzone boundary. There is therefore a limit to how small $h$ can be made before diffraction dominates the dispersion. Since proximity to the Brillouin-zone boundary will result in a mode with a short propagation distance, one should look to optimize the divergence of the dispersion relation from the
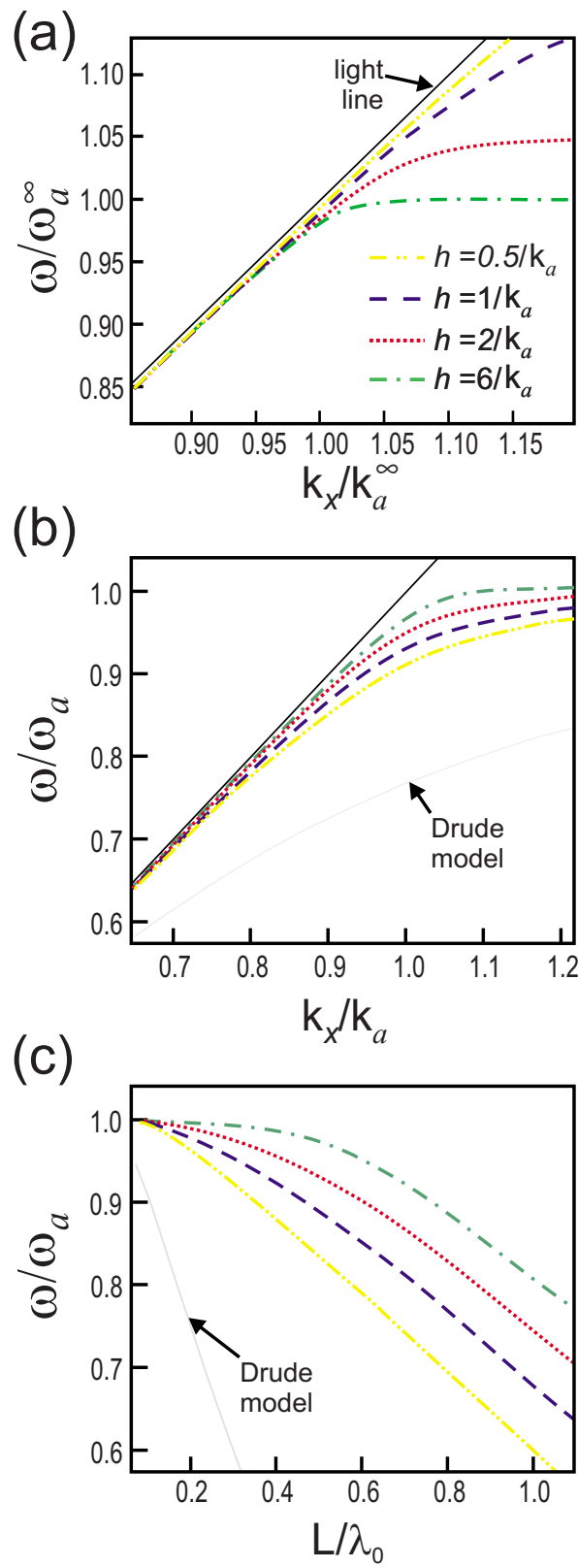

FIG. 4. (Color online) (a) The dispersion obtained from Eq. (21) for a hole period defined by $d=1.1 / k_{a}^{\infty}$ (i.e., a Brillouin-zone boundary occurring at $\left.k_{x} / k_{a}^{\infty}=2.86\right)$ and $\varepsilon_{h}=10$, varying the depth of the hole depth $h$. The frequency and wave vector are normalized using $\omega_{a}^{\infty}$ [Eq. (15)] and $k_{a}^{\infty}$ [Eq. (16)], respectively. (b) The same dispersion relations as in (a) normalized by the asymptotic frequency for finite depth holes, $\omega_{a}$ and corresponding wave vector, $k_{a}$, allowing us to compare the curvature of dispersion relations with different asymptotic frequencies. For comparison, the normalized SP dispersion at the interface between vacuum and an unstructured metal surface is shown (thin gray line). In both (a) and (b), higher order surface modes, above the asymptotic frequency, have been left out for clarity. (c) Scaled evanescent decay length normal to the vacuum-surface interface, $\mathbf{L} / \lambda_{0}$, for the modes in (a) and (b).

light line well away from the Brillouin-zone boundary. This essentially results in an optimum hole depth $h$ for a given structure. ${ }^{15}$ It is clear that the limits described above mean that there are restrictions to the tunability of a designer SP. 
Indeed, for a surface defined by $\varepsilon_{h}=10$ and $d=1.1 / k_{a}^{\infty}$, the hole depth $h=0.5 / k_{a}^{\infty}$ represents the approximate lower limit for $h$, or a "best case," giving the largest divergence from the light line for a low loss, nondiffractive mode. ${ }^{15}$

For comparison, we also plot in Fig. 4(b) the normalized dispersion characteristics of a SP on a flat, unstructured metal surface, calculated assuming a lossless Drude model permittivity. ${ }^{16}$ The plasma frequency of a typical metal lies in the UV region of the electromagnetic spectrum, and the dispersion of a SP mode on a metal diverges from the light line in the visible spectral range. ${ }^{17}$ The electromagnetic surface modes described in the previous sections are often referred to as designer (or spoof) $\mathrm{SPs},{ }^{3}$ due to the similarity in dispersion to that of a true SP at the interface between vacuum and a flat, real metal surface. However, it is clear from Fig. 4(b) that even our best case designer SP cannot match the dispersion characteristics of a SP on a real metal surface.

\section{Confinement of surface modes}

The capability to confine electromagnetic energy at the interface of a conductor makes these designer SPs very promising for applications where efficient guiding of electromagnetic radiation on very small length scales is important. Good subwavelength confinement is therefore encouraging for the realization of surfaces for applications such as subwavelength microscopy, non-inear optics, photolithography, and sensing.

Below the asymptotic frequency of the dispersion relation, the electric-field strength of the surface mode will decay exponentially in the direction normal to the vacuummetal interface, with a decay length $L$ given by

$$
\frac{1}{L^{2}}=k_{x}^{2}+k_{y}^{2}-\left(\frac{\omega}{c}\right)^{2} .
$$

A mode characterized by $\sqrt{k_{x}^{2}+k_{y}^{2}} \gg \frac{\omega}{c}$ will be strongly confined to the surface. In Fig. 4(c) we plot the exponential decay length $L$ for the modes in Figs. 4(a) and 4(b). Note that we normalize $L$ by the vacuum wavelength of light $\lambda_{0}$ $=2 \pi c / \omega$. It is clear from Fig. 4(c) that one can obtain strong subwavelength confinement for a designer SP mode: for the best case dispersion described in Sec. III C (i.e., for a surface defined by $d=1.1 / k_{a}^{\infty}, h=0.5 / k_{a}^{\infty}$, and $\left.\varepsilon_{h}=10\right)$, at $\omega=0.95 \omega_{a}$ we have $L \sim 0.2 \lambda_{0}$.

We can compare this best case confinement (yellow, dotdot-dash line in Fig. 4) to that for a surface defined by $a$ $=75 \mu \mathrm{m}, d=100 \mu \mathrm{m}, h=25 \mu \mathrm{m}$, and $\varepsilon_{h}=1$, reported recently as $L \sim 0.5 \lambda_{0} .{ }^{8,10}$ Moreover, it should be noted that the surface considered in Refs. 8 and 10 is highly diffractive (indeed the reported value of $L \sim 0.5 \lambda_{0}$ corresponds to the confinement at the Brillouin-zone boundary). For applications, one would ideally like to have strong-mode confinement for wave vectors well below the Brillouin-zone boundary and for frequencies well below the asymptotic frequency in order to limit propagation losses. ${ }^{7}$ This essentially limits the range over which one can "tune" the dispersion of the SP-like mode: stronger confinement may be achieved via a decrease in $d$, which is ultimately limited by the hole size $a$, while an optimum depth $h$ for a given structure exists which results in the strongest confinement for a nondiffracting structure.

For comparison, we also plot in Fig. 4(c) the field confinement expected for a SP on a flat, unstructured metal surface, calculated assuming a lossless Drude model dielectric function. ${ }^{16}$ It is clear that even our best case designer SP cannot match the confinement characteristics of a SP on a real metal surface.

\section{CONCLUSIONS}

To conclude: by employing a modal matching method, we have derived a completely general and full analytical formalism for determining the dispersion of the surface electromagnetic modes at the interface between vacuum and a substrate characterized by a square array of holes in a perfect conductor. Previously, García de Abajo and Sáenz ${ }^{5,12}$ demonstrated that the inclusion of higher order waveguide modes for this structure generates a dispersion relation which, in the longwavelength limit, lies significantly closer to the light line than that predicted earlier by Pendry and co-workers. 3,4 While higher order waveguide modes are clearly important in the long-wavelength limit, we show here that diffracted evanescent waves in the region above the holes modify considerably the dispersion of surface modes near the asymptotic cutoff frequency. The simple dispersion relation derived here, which takes into account these evanescent diffracted orders, shows very good agreement with experimentally measured dispersion curves in the microwave frequency region. Finally, we compare our analytical dispersion relation for designer SPs on dimpled surfaces to that of a real SP at a metal-vacuum interface, and show that designer SPs cannot match the confinement characteristics of SPs on a real metal surface.

\section{ACKNOWLEDGMENTS}

The authors would like to thank M. J. Lockyear and W. L. Barnes for helpful discussions and acknowledge the financial support of the EPSRC-GB, while E.H. acknowledges support from the RCUK.
*FAX: +44 1392 264111; e.hendry@exeter.ac.uk
${ }^{1}$ R. Ulrich and M. Tacke, Appl. Phys. Lett. 22, 251 (1973).
${ }^{2}$ A. F. Harvey, IRE Trans. Microwave Theory Tech. 8, 30 (1960).
${ }^{3}$ J. B. Pendry, L. Martin-Moreno, and F. J. Garcia-Vidal, Science
305, 847 (2004).

${ }^{4}$ F. J. Garcia-Vidal, L. Martin-Moreno, and J. B. Pendry, J. Opt. A, Pure Appl. Opt. 7, S97 (2005).

${ }^{5}$ F. J. Garcia de Abajo and J. J. Sáenz, Phys. Rev. Lett. 95, 
233901 (2005).

${ }^{6}$ Y. C. Lan and R. L. Chern, Opt. Express 14, 11339 (2006).

${ }^{7}$ L. Shen, X. Chen, and T. J. Yang, Opt. Express 16, 3326 (2008).

${ }^{8}$ S. A. Maier and S. R. Andrews, Appl. Phys. Lett. 88, 251120 (2006).

${ }^{9}$ A. P. Hibbins, B. R. Evans, and J. R. Sambles, Science 308, 670 (2005).

${ }^{10}$ C. R. Williams, S. R. Andrews, S. A. Maier, A. I. FernándezDomínguez,L. Martín-Moreno, and F. J. García-Vidal, Nat. Photonics 2, 175 (2008).

${ }^{11}$ R. C. McPhedran, G. H. Derrick, and L. C. Botten, in Electromagnetic Theory of Gratings, edited by R. Petit (SpringerVerlag, Berlin, 1980).

${ }^{12}$ F. J. García de Abajo, Rev. Mod. Phys. 79, 1267 (2007).

${ }^{13}$ A. P. Hibbins, E. Hendry, M. J. Lockyear, and J. R. Sambles, Opt. Express 16, 20441 (2008).
${ }^{14}$ The asymptotic frequency for the dispersion $\omega_{a}$ and corresponding wave vector, $k_{a}$, cannot be found using Eq. (18). Instead, we find the asymptotic frequency by fully solving the dispersion relation in Eq. (21): $\omega_{a}\left(k_{a}\right)$ is found to be $2.15 \omega_{a}^{\infty}\left(2.15 k_{a}^{\infty}\right)$, $1.49 \omega_{a}^{\infty}\left(1.49 k_{a}^{\infty}\right), 1.18 \omega_{a}^{\infty}\left(1.18 k_{a}^{\infty}\right)$, and $1.01 \omega_{a}^{\infty}\left(1.01 k_{a}^{\infty}\right)$ for surfaces defined by $h=0.5 / k_{a}^{\infty}, h=1 / k_{a}^{\infty}, 2 / k_{a}^{\infty}$, and $6 / k_{a}^{\infty}$, respectively.

${ }^{15}$ For a sample defined by $d=1.1 / k_{a}^{\infty}$ and $\varepsilon_{h}=10$ (corresponding to a hole size $a=0.9 d) h$ should not be made significantly smaller than $\sim 0.5 / k_{a}^{\infty}$ (corresponding to a hole depth $h=0.5 d$ ). For this value of $\varepsilon_{h}, h<0.5 / k_{a}^{\infty}$ corresponds to $k_{a}>2.15 k_{a}^{\infty}$, i.e., the region of divergent dispersion will tend towards the Brillouin-zone boundary at $2.86 k_{a}^{\infty}$.

${ }^{16}$ W. L. Barnes, J. Opt. A, Pure Appl. Opt. 8, S87 (2006).

${ }^{17}$ W. L. Barnes, A. Dereux, and T. W. Ebbesen, Nature (London) 424, 824 (2003). 